\title{
Study of antimicrobial resistance pattern in blood isolates from critical care unit at a Tertiary Care hospital, Udaipur, Rajasthan
}

\author{
Ritu Bhatnagar', Pragnesh Patel ${ }^{2, *}$ \\ Assistant Professor, Dept. of Microbiology, Pacific Medical College and Hospital, Pacific Medical University, Udaipur, \\ Rajasthan, India
}

*Corresponding Author:

Email: dr.pt185@gmail.com

\begin{abstract}
Introduction: Microorganisms present in circulating blood are a threat to the every organ of the body. Blood stream infections can have serious consequences and timely detection and identification of blood stream pathogen is one of the most important functions of microbiology laboratory. Positive blood culture helps in providing a specific etiological diagnosis and antimicrobial susceptibility pattern.

The present study was undertaken to find the pattern of etiological agents of BSI \& their antimicrobial susceptibility.

Materials and Methods: A study was carried out from July 2016 to December 2017 at a tertiary care hospital, Udaipur, Rajasthan, India using conventional blood culture method. Organisms were identified by standard microbiological procedures and antibiotic sensitivity was performed using the kirby-bauer disk diffusion method.

Result and Discussion: A total of 800 samples of suspected blood stream infections (BSI) were included during the study period. Bacterial growth was obtained in 299 samples (37.37\%). Gram-positive cocci accounted for 53.16\% and Gram negative for $46.50 \%$ and candida species as $0.33 \%$ of positive cultures. Coagulase negative staphylococcus $(38.12 \%)$, Staphylococcus aureus (13.04\%), Pseudomonas species (13.71\%) and Klebsiella species were predominant organisms. The antimicrobial susceptibility pattern of blood isolates showed high resistance to routinely used antimicrobial agents.

Conclusion: This emphasizes the importance of institutional antibiotic policy, stringent infection control practices and judicious use of antibiotics.
\end{abstract}

Keywords: BSI, Antimicrobial resistance, Blood isolates.

\section{Introduction}

Microorganisms present in the circulating blood whether continuously, intermittently or transiently are a threat to every organ of the body. Microbial invasion of the blood stream can have serious immediate consequences including shock, multiple organ failure, disseminated intravascular coagulation (DIC) and death. So timely detection and identification of blood borne pathogen is one of the most important functions of the microbiology laboratory. ${ }^{1}$

Increasing antimicrobial resistance among blood stream infections have been reported in many studies conducted in India and other countries worldwide. ${ }^{2}$

\section{Aim}

The present study was conducted in our set up to find out the pattern of etiological agent and their antimicrobial sensitivity to guide the clinician towards prevalent pathogen and proper selection of antibiotics for the treatment of patients admitted in the hospital and nosocomial blood stream infections

\section{Material and Methods}

The study was performed from July 2016 to December 2017. Samples were collected from suspected patients admitted in Critical Care Unit with signs and symptoms of BSI at a tertiary care hospital, Udaipur, Rajasthan, India.

During the study period, $5-10 \mathrm{ml}$ blood from each adult patient was taken and inoculated in blood culture

bottle. $50 \mathrm{ml}$ brain heart infusion broth media (Hi media) was used in bottle. Bottles were incubated aerobically for 7 days, in an incubator at $37^{\circ} \mathrm{C}$ and subculture were done on Nutrient agar, MacConkey agar \& blood agar plates. After 48 hours and 7 days, in between these time points subcultures were done if visible turbidity was found.

Bacterial growth was identified up-to species level by morphology and conventional biochemical tests using standard protocols. ${ }^{3}$

Antimicrobial susceptibility testing was performed by disc diffusion method (Kirby-Bauer method) according to CLSI guidelines. ${ }^{4}$

Antifungal susceptibility testing was not done.

\section{Result}

Total 800 test samples were analyzed. Out of that in 299 cases, bacterial pathogens were isolated. Organisms, isolated from the blood samples were Gram-positive cocci, Gram-negative bacilli and candida species. 
Table 1: distribution of isolates obtained from blood culture samples

\begin{tabular}{|c|l|c|c|}
\hline S. No. & \multicolumn{1}{|c|}{ Isolates } & Total No. & $\%$ \\
\hline 1 & CONS & 114 & 38.12 \\
\hline 2 & S. aureus & 39 & 13.04 \\
\hline 3 & Entero cocci & 5 & 1.67 \\
\hline 4 & S. viridans & 1 & 0.33 \\
\hline TOTAL GPC & Pseudomonas & 159 & 53.16 \\
\hline 1. & Klebsiella & 39 & 13.71 \\
\hline 2. & E. Coli & 29 & 13.04 \\
\hline 3. & Acinetobacter & 25 & 9.70 \\
\hline 4. & Enterobacter & 3 & 8.36 \\
\hline 5. & Salmonella & 2 & 0.70 \\
\hline \multicolumn{2}{|c|}{6.} & 139 & 46.50 \\
\hline TOTAL GNB & Candida & 1 & 0.33 \\
\hline \multicolumn{2}{|c|}{7.} & $299 / 800$ & \\
\hline TOTAL & & $37.37 \%$ & \\
\hline Blood culture Positivity $(\%)$ & & \\
\hline
\end{tabular}

Most common Gram positive isolates were CONS (38.12\%), followed by Staphylococcus aureus (13.04\%), Enterococcus species (1.67\%) and Streptococcus viridans (0.33\%), as shown in Fig. 1.

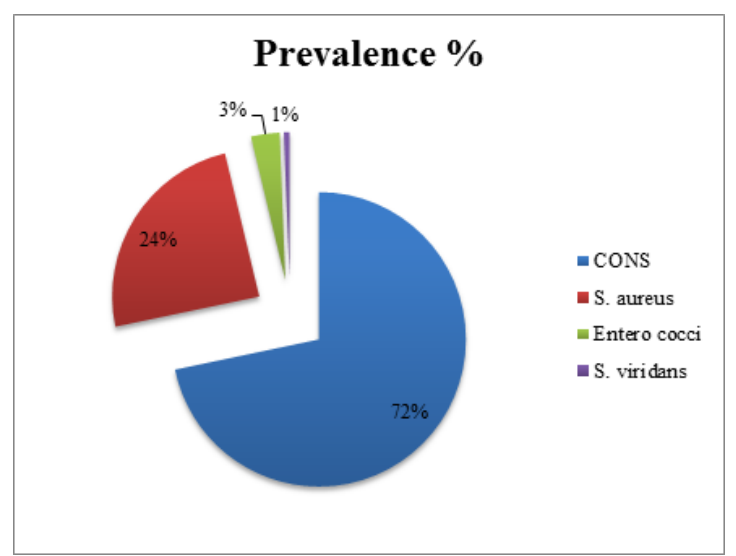

Fig 1: Prevalence of various Gram positive isolates from blood culture

Most common Gram negative isolates were Pseudomonas species (13.71\%), followed by Klebsiella species (13.04\%), E. coli $(9.70 \%)$, Acinetobacter species (8.36\%), Enterobacter species (1\%) and Salmonella Typhi $(0.70 \%)$. as shown in Fig. 2.

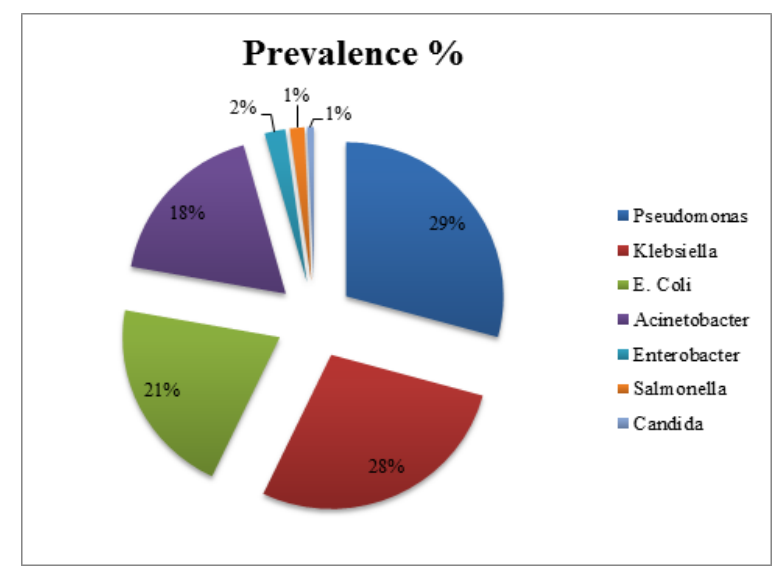

Fig 2: Prevalence of various Gram-negative isolates from blood culture 
Among 159 Gram positive and 139 Gram negative isolates, different patterns of antimicrobial resistance were observed as shown in table 2 and table 3 respectively.

Table 2: Antimicrobial resistance (\%) of various Gram positive isolates

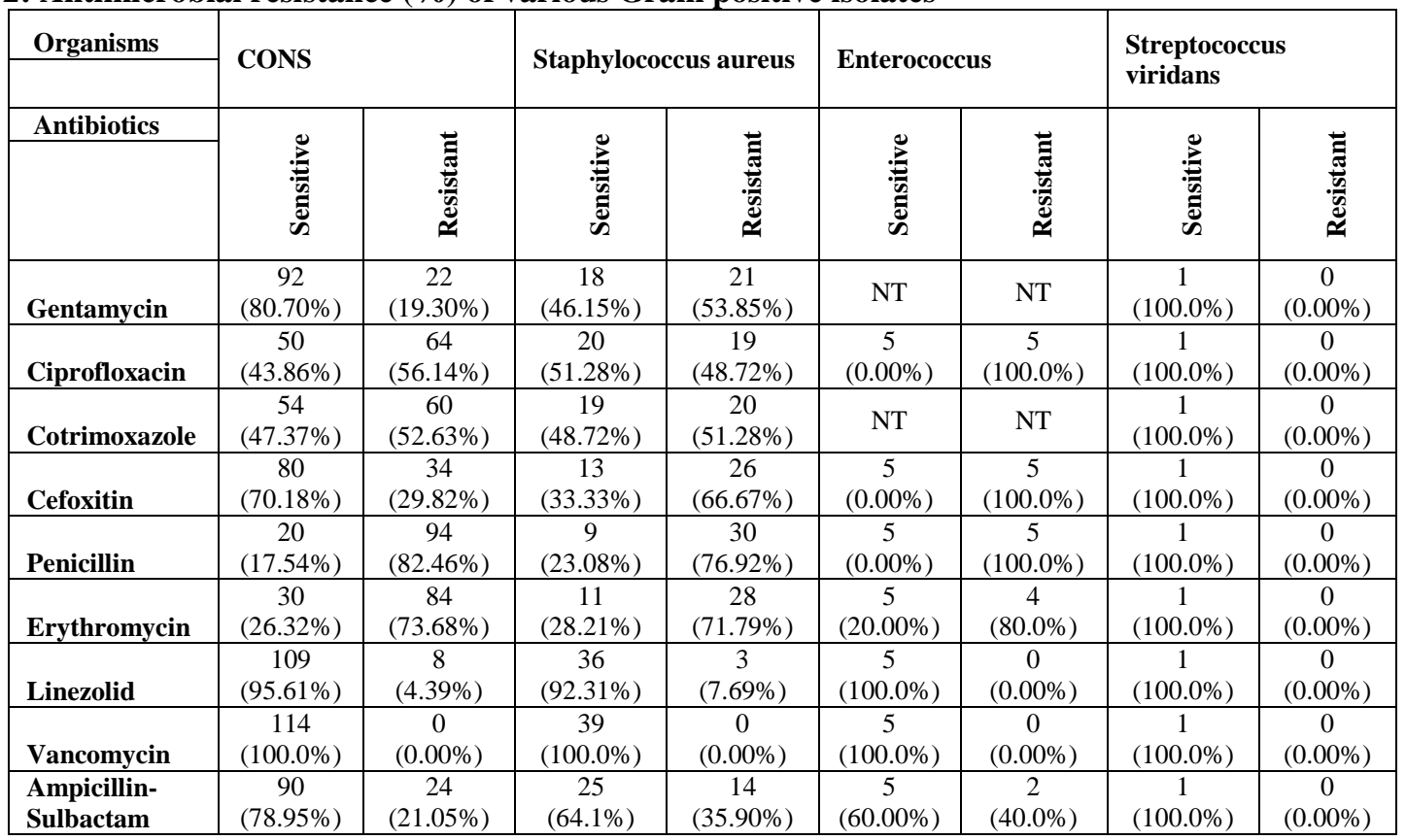

In Gram-positive isolates, high resistance to different antibiotics was observed in CONS and Staphylococcus aureus.

CONS showed highest resistance to penicillin (82.46\%), followed by Erythromycin (73.68\%), Ciprofloxacin $(56.14 \%)$ and cotrimoxazole (52.63\%). In Staphylococcus aureus, resistance to penicillin was (76.92\%), Erythromycin (71.79\%), cotrimoxazole $(51.28 \%)$ and Gentamycin $(53.85 \%)$. CONS showed good sensitivity to Vancomycin (100\%), followed by Linezolid (95.61\%), Gentamycin (80.70\%) and Ampicillin-sulbactam(78.95\%).

Staphylococcus aureus showed good sensitivity to Vancomycin (100\%), followed by Linezolid (92.31\%), Ampicillin-sulbactam(64.1\%) 
Table 3: Antimicrobial resistance (\%) of various Gram-negative isolates

\begin{tabular}{|c|c|c|c|c|c|c|c|c|c|c|c|c|}
\hline \multirow{2}{*}{$\begin{array}{c}\text { Organisms } \\
\text { Antibiotics }\end{array}$} & \multicolumn{2}{|c|}{ Pseudomonas } & \multicolumn{2}{|c|}{ Klebsiella } & \multicolumn{2}{|c|}{ E.coli } & \multicolumn{2}{|c|}{ Acinetobacter } & \multicolumn{2}{|c|}{ Enterobacter } & \multicolumn{2}{|c|}{ Salmonella sp } \\
\hline & Sensitive & Resistant & Sensitive & Resistant & Sensitive & Resistant & Sensitive & Resistant & Sensitive & Resistant & Sensitive & Resistant \\
\hline Imipenem & $\begin{array}{c}41 \\
(100.0 \%) \\
\end{array}$ & $\begin{array}{c}0 \\
(0.00 \%) \\
\end{array}$ & $\begin{array}{c}39 \\
(100.0 \%) \\
\end{array}$ & $\begin{array}{c}0 \\
(0.00 \%) \\
\end{array}$ & $\begin{array}{c}29 \\
(100.0 \%)\end{array}$ & $\begin{array}{c}0 \\
(0.00 \%) \\
\end{array}$ & $\begin{array}{c}25 \\
(100.0 \%)\end{array}$ & $\begin{array}{c}0 \\
(0.00 \%) \\
\end{array}$ & $\begin{array}{c}3 \\
(100.0 \%) \\
\end{array}$ & $\begin{array}{c}0 \\
(0.00 \%) \\
\end{array}$ & $\begin{array}{c}2 \\
(100.0 \%)\end{array}$ & $\begin{array}{c}0 \\
(0.00 \%)\end{array}$ \\
\hline $\begin{array}{l}\text { Ampicillin- } \\
\text { Sulbactam } \\
\end{array}$ & $\begin{array}{c}10 \\
(24.39 \%) \\
\end{array}$ & $\begin{array}{c}31 \\
(75.61 \%) \\
\end{array}$ & $\begin{array}{c}9 \\
(58.62 \%) \\
\end{array}$ & $\begin{array}{c}30 \\
(76.92 \%) \\
\end{array}$ & $\begin{array}{c}17 \\
(58.62 \%) \\
\end{array}$ & $\begin{array}{c}12 \\
(41.38 \%) \\
\end{array}$ & $\begin{array}{c}6 \\
(24.00 \%) \\
\end{array}$ & $\begin{array}{c}19 \\
(76.00 \%) \\
\end{array}$ & $\begin{array}{c}2 \\
(66.67 \%) \\
\end{array}$ & $\begin{array}{c}1 \\
(33.33 \%) \\
\end{array}$ & $\begin{array}{c}2 \\
(100.0 \%) \\
\end{array}$ & $\begin{array}{c}0 \\
(0.00 \%) \\
\end{array}$ \\
\hline Amikacin & $\begin{array}{c}22 \\
(53.66 \%) \\
\end{array}$ & $\begin{array}{c}19 \\
(46.34 \%) \\
\end{array}$ & $\begin{array}{c}17 \\
(51.72 \%) \\
\end{array}$ & $\begin{array}{c}22 \\
(56.41 \%) \\
\end{array}$ & $\begin{array}{c}15 \\
(51.72 \%) \\
\end{array}$ & $\begin{array}{c}14 \\
(48.28 \%) \\
\end{array}$ & $\begin{array}{c}5 \\
(20.00 \%) \\
\end{array}$ & $\begin{array}{c}20 \\
(80.00 \%) \\
\end{array}$ & $\begin{array}{c}3 \\
(100.0 \%) \\
\end{array}$ & $\begin{array}{c}0 \\
(0.00 \%) \\
\end{array}$ & $\begin{array}{c}2 \\
(100.0 \%) \\
\end{array}$ & $\begin{array}{c}0 \\
(0.00 \%) \\
\end{array}$ \\
\hline Gentamycin & $\begin{array}{c}16 \\
(39.02 \%)\end{array}$ & $\begin{array}{c}25 \\
(60.98 \%)\end{array}$ & $\begin{array}{c}14 \\
(44.83 \%)\end{array}$ & $\begin{array}{c}25 \\
(64.10 \%)\end{array}$ & $\begin{array}{c}13 \\
(44.83 \%)\end{array}$ & $\begin{array}{c}16 \\
(55.17 \%)\end{array}$ & $\begin{array}{c}8 \\
(32.00 \%)\end{array}$ & $\begin{array}{c}17 \\
(68.00 \%)\end{array}$ & $\begin{array}{c}3 \\
(100.0 \%)\end{array}$ & $\begin{array}{c}0 \\
(0.00 \%)\end{array}$ & $\begin{array}{c}1 \\
(50.00 \%)\end{array}$ & $\begin{array}{c}1 \\
(50.00 \%)\end{array}$ \\
\hline Ciprofloxacin & $\begin{array}{c}15 \\
(36.59 \%)\end{array}$ & $\begin{array}{c}26 \\
(63.41 \%)\end{array}$ & $\begin{array}{c}10 \\
(62.07 \%)\end{array}$ & $\begin{array}{c}29 \\
(74.36 \%)\end{array}$ & $\begin{array}{c}18 \\
(62.07 \%)\end{array}$ & $\begin{array}{c}11 \\
(37.93 \%)\end{array}$ & $\begin{array}{c}5 \\
(20.00 \%)\end{array}$ & $\begin{array}{c}20 \\
(80.00 \%)\end{array}$ & $\begin{array}{c}2 \\
(66.67 \%)\end{array}$ & $\begin{array}{c}1 \\
(33.33 \%)\end{array}$ & $\begin{array}{c}2 \\
(100.0 \%)\end{array}$ & $\begin{array}{c}0 \\
(0.00 \%)\end{array}$ \\
\hline Cefixime & $\begin{array}{c}1 \\
(2.44 \%) \\
\end{array}$ & $\begin{array}{c}40 \\
(97.56 \%) \\
\end{array}$ & $\begin{array}{c}8 \\
(48.28 \%) \\
\end{array}$ & $\begin{array}{c}31 \\
(79.49 \%) \\
\end{array}$ & $\begin{array}{c}14 \\
(48.28 \%) \\
\end{array}$ & $\begin{array}{c}15 \\
(51.72 \%) \\
\end{array}$ & $\begin{array}{c}1 \\
(4.00 \%)\end{array}$ & $\begin{array}{c}24 \\
(96.00 \%) \\
\end{array}$ & $\begin{array}{c}2 \\
(66.67 \%) \\
\end{array}$ & $\begin{array}{c}1 \\
(33.33 \%) \\
\end{array}$ & $\begin{array}{c}2 \\
(100.0 \%) \\
\end{array}$ & $\begin{array}{c}0 \\
(0.00 \%) \\
\end{array}$ \\
\hline Cefotaxime & $\begin{array}{c}12 \\
(29.27 \%) \\
\end{array}$ & $\begin{array}{c}29 \\
(70.73 \%) \\
\end{array}$ & $\begin{array}{c}9 \\
(68.97 \%) \\
\end{array}$ & $\begin{array}{c}30 \\
(76.92 \%) \\
\end{array}$ & $\begin{array}{c}20 \\
(68.97 \%) \\
\end{array}$ & $\begin{array}{c}9 \\
(31.03 \%) \\
\end{array}$ & $\begin{array}{c}4 \\
(16.00 \%)\end{array}$ & $\begin{array}{c}21 \\
(84.00 \%)\end{array}$ & $\begin{array}{c}3 \\
(100.0 \%)\end{array}$ & $\begin{array}{c}0 \\
(0.00 \%)\end{array}$ & $\begin{array}{c}2 \\
(100.0 \%)\end{array}$ & $\begin{array}{c}0 \\
(0.00 \%)\end{array}$ \\
\hline Cotrimoxazole & $\begin{array}{c}2 \\
(4.88 \%) \\
\end{array}$ & $\begin{array}{c}39 \\
(95.12 \%) \\
\end{array}$ & $\begin{array}{c}5 \\
(65.52 \%) \\
\end{array}$ & $\begin{array}{c}34 \\
(87.18 \%) \\
\end{array}$ & $\begin{array}{c}19 \\
(65.52 \%) \\
\end{array}$ & $\begin{array}{c}10 \\
(34.48 \%) \\
\end{array}$ & $\begin{array}{c}0 \\
(0.00 \%) \\
\end{array}$ & $\begin{array}{c}25 \\
(100.0 \%) \\
\end{array}$ & $\begin{array}{c}2 \\
(66.67 \%) \\
\end{array}$ & $\begin{array}{c}1 \\
(33.33 \%) \\
\end{array}$ & $\begin{array}{c}1 \\
(50.00 \%) \\
\end{array}$ & $\begin{array}{c}1 \\
(50.00 \%) \\
\end{array}$ \\
\hline Ceftazidime & $\begin{array}{c}11 \\
(26.83 \%) \\
\end{array}$ & $\begin{array}{c}30 \\
(73.17 \%) \\
\end{array}$ & $\begin{array}{c}8 \\
(58.62 \%) \\
\end{array}$ & $\begin{array}{c}31 \\
(79.49 \%) \\
\end{array}$ & $\begin{array}{c}17 \\
(58.62 \%) \\
\end{array}$ & $\begin{array}{c}12 \\
(41.38 \%) \\
\end{array}$ & $\begin{array}{c}7 \\
(28.00 \%) \\
\end{array}$ & $\begin{array}{c}18 \\
(72.00 \%) \\
\end{array}$ & $\begin{array}{c}1 \\
(33.33 \%) \\
\end{array}$ & $\begin{array}{c}2 \\
(66.67 \%) \\
\end{array}$ & $\begin{array}{c}2 \\
(100.0 \%) \\
\end{array}$ & $\begin{array}{c}0 \\
(0.00 \%) \\
\end{array}$ \\
\hline $\begin{array}{l}\text { Piperacillin- } \\
\text { Tazobactem }\end{array}$ & $\begin{array}{c}29 \\
(70.73 \%) \\
\end{array}$ & $\begin{array}{c}12 \\
(29.27 \%) \\
\end{array}$ & $\begin{array}{c}25 \\
(68.97 \%) \\
\end{array}$ & $\begin{array}{c}14 \\
(35.90 \%) \\
\end{array}$ & $\begin{array}{c}20 \\
(68.97 \%) \\
\end{array}$ & $\begin{array}{c}9 \\
(31.03 \%) \\
\end{array}$ & $\begin{array}{c}14 \\
(56.00) \% \\
\end{array}$ & $\begin{array}{c}11 \\
(44.00 \%) \\
\end{array}$ & $\begin{array}{c}3 \\
(100.0 \%) \\
\end{array}$ & $\begin{array}{c}0 \\
(0.00 \%) \\
\end{array}$ & $\begin{array}{c}2 \\
(100.0 \%) \\
\end{array}$ & $\begin{array}{c}0 \\
(0.00 \%) \\
\end{array}$ \\
\hline
\end{tabular}


In Gram negative isolates, Pseudomonas species showed highest resistance for Cefixime $(97.56 \%)$ and cotrimoxazole $(95.12 \%)$ and good sensitivity for Imipenem (100\%), piperacillin-tazobactem $(70.73 \%)$ and amikacin $(53.66 \%)$

Klebsiella species showed high resistance to cotrimoxazole $(87.18 \%)$, ceftazidime $(79.49 \%)$ and cefixime (79.49\%), and good sensitivity for Imipenem (100\%), followed by piperacillin-tazobactem $(64.10 \%)$

\section{Discussion}

During the study period, among $53.16 \%$ GPC isolates, Coagulase negative staphylococci (CONS) were $38.12 \%$ and Staphylococcus aureus were $13.04 \%$ (as shown in Table 1). Similar observations were reported from Arora $\mathrm{U}^{5} \mathrm{P}$ jyothi $^{6}$ an T swami. ${ }^{7}$

Among $\quad 46.50 \%$ Gram-negative isolates, Pseudomonas $(13.71 \%)$ and Klebsiella species (13.04\%) were predominant. Positivity of Pseudomonas species in blood culture was quite high in present study and positivity of Klebsiella species was similar as compared to other studies. Anu Gupta, ${ }^{8}$ Kalpesh Gohel. ${ }^{9}$

In the present study, antimicrobial resistance patterns of all GPC were showing increasing resistance pattern to almost all the antibiotics routinely used. GPC were showing high resistance to penicillin $(82.46 \%)$, followed by Erythromycin (73.68\%), Ciprofloxacin (56.14\%) and cotrimoxazole (52.63\%), but most of the isolates were susceptible to vancomycin \& linezolid (zero resistance pattern). ${ }^{7}$

We found GNB resistant to Cefixime $(97.56 \%)$ and cotrimoxazole $(95.12 \%)$ and good sensitivity for Imipenem (100\%), piperacillin-tazobactem (70.73\%) and amikacin $(53.66 \%)$, Similar observations were made by other studies.

During the study period, all isolates followed resistance pattern to commonly prescribed antibiotics and newer generation of drugs also.

This shows the narrow range of antimicrobial choice for the treatment of blood stream infections which can be life threatening.

Irrational use of powerful antibiotics for prolonged period with compromised host conditions might be responsible for emergence of multi drug resistant strains.

\section{Conclusion}

The study emphasizes the importance of rationale antibiotic prescription by clinicians and stringent infection control policy by institute to prevent emerging drug resistance and also the need for development of new drugs and vaccines.

\section{References}

1. Betty A. Forbes, Daniel F. Sahm, Alice S. Weissfeld, William Robert Bailey. Blood stream Infections. Bailey $\&$ Scott's diagnostic microbiology. $13^{\text {th }}$ edition. Elsevier
Health Sciences. 2014.

2. Madsen KM, Schonheydr HC, Kristensen B, Sorensen HT. Secular trends in incidence and mortality of bacteraemia in a Danish country 1981-1994. Acta Pathol Microbiol Immunol Scand 1999;107:346-52.

3. Collee JG, Miles RS, Watt B. Tests for identification of bacteria In: Mackie and Mc Cartney's Practical Medical Microbiology (Collee JG, Fraser AG, Marmion BP, Simmons A, eds.), 14 edition, London: Churchill Livingstone, 1996;131-45.

4. Clinical and laboratory standards Institute (CLSI). Performance standards for antimicrobial susceptibility testing: $21^{\text {st }}$ Informational supplement 2011. CLSI document $\mathrm{M}_{100}-\mathrm{S}_{20}$ wayne. PA: CLSI: 2011.

5. Arora U, Devi P. Bacterial profile of blood stream infections and antibiotic resistance pattern of isolates. $J K$ Science 2007;9(4):186-90.

6. Jyothi P, Basavaraj MC, Basavaraj PV. Bacteriological profile of neonatal septicemia and antibiotic susceptibility pat- tern of the isolates. J Nat Sc Biol Med 2013;4:306-9.

7. Taruna Swami, Punit Sharma, Anjali Gupta, B P Sharma. Trends of in-vitro antimicrobial resistance pattern in blood isolates in a tertiary care institute in North-west region of Rajasthan over a period of 3 years. J. Commun.Dis. 2014;46(3):44-6.

8. Gupta A, Sharma S, Arora A, Gupta A. Changing trends of in vitro antimicrobial resistance pattern in blood isolates in a tertiary care hospital over a period of 4 years. Ind J Med Sci 2010; 64(11):485- 92.

9. Kalpesh Gohel, Amit Jojera, Shailesh Soni, Sishir Gang, Ravindra Sabnis, and Mahesh Desai. Bacteriological Profile and Drug Resistance Patterns of Blood Culture Isolates in a Tertiary Care Nephrourology Teaching Institute. BioMed Research International 2014.

Abbrevations: BSI - Blood stream infections, CONSCoagulase negative staphylococcus.

How to cite this article: Bhatnagar R, Patel P. Study of antimicrobial resistance pattern in blood isolates from critical care unit at a Tertiary Care hospital, Udaipur, Rajasthan. Indian J Microbiol Res 2018;5(1):61-65. 